\title{
Nuclear Magnetic Resonance and Acoustic Emission on Initial Crack and Fracture Development of Coal with Different Strengths
}

\author{
Jun Han ${ }^{1,2}$, Luyu Cui ${ }^{1}$, Qianjia Hui ${ }^{1, *}$ and Han Liang ${ }^{1}$ \\ ${ }^{1}$ College of Mining, Liaoning Technical University, Fuxin 123000, China \\ ${ }^{2}$ Liaoning Province Engineering Research Center for Safety Mining and Clean Utilization of Coal Resources, Fuxin 123000, China
}

Received 9 March 2021; Accepted 28 June 2021

\begin{abstract}
The initial fracture and development of fracture under loading conditions of coal are closely related to the stability of surrounding rock and geo-dynamic disasters. In this study, nuclear magnetic resonance (NMR), acoustic emission (AE) and 3D-CAD reduction techniques were employed to analyze the initial fracture distribution, the fracture development and failure mode of coal samples with different strengths under uniaxial loading. Based on NMR and 3D-CAD reduction, the initial fracture rate and fracture distribution of coal samples were obtained. Through uniaxial loading and AE, the distribution of fracture points was analysed. The dominated fracture of the failure state was reconstructed using 3D-CAD. Results show the initial crack rate of hard coal is low, and the ringing accounts and AE energy are small in the initial stage of loading. However, they increase rapidly in the later stage of loading, showing the characteristics of brittleness. In the initial state, the fracture rate of soft coal sample is higher than that of hard coal, and the growth of fracture point is fast. In the later stage, the fracture point number and AE energy are similar to that of hard coal. The initial fracture rate and fracture distribution of coal with different strengths are different, resulting in different occurrence and development of secondary fractures in loading state, which indicates that the deformational behaviour, failure mode and energy release are different for coal with different strengths. This study provides a theoretical basis for the stability analysis and geodynamic disasters prevention of coal with different strengths.
\end{abstract}

Keywords: Coal, Crack, Fracture, Energy, Acoustic emission

\section{Introduction}

From the perspective of mining engineering, the structural instability of coal and rock mass and geological dynamic disasters such as rockburst are closely related to the bearing capacity, failure mode and energy accumulation within the coal mass. As coal is a discontinuous medium composed by matrix and fractures, the distribution of the primary cracks and the fracture development under loading conditions are the keys for the failure characteristics and energy evolution of the coal mass [1]. Therefore, it is of great significance to study the distribution of initial coal cracks and the fracture development for the stability of surrounding rock and the prevention of geo-disasters.

Nuclear magnetic resonance (NMR) technology is a physical process in which the nuclear spin energy levels are Zeeman separated by an external magnetic field on the atomic nucleus with non-zero nuclear magnetic moment, and the $\mathrm{RF}$ radiation of a specific frequency is absorbed by resonance. At present, NMR technology is used in the rock mechanics to test the hydrogen atoms in rock fractures to obtain the distribution of internal fractures [2]. NMR does not damage the structure of rock, so, Mechanical tests can be conducted after NMR testing.

Acoustic emission (AE) technology is a method to obtain the damage and crack position in materials based on the emission and detection of elastic waves. AE technology

*E-mail address: hqi026@126.com

ISSN: 1791-2377 @ 2021 School of Science, IHU. All rights reserved.

doi:10.25103/iestr.143.21 can accurately determine the damage position of materials in the process of dynamic loading. The AE testing can also measure the energy change of the sample in the loading process. In recent years, as a non-destructive testing technology, AE is widely used in the field of mining engineering [3-4].

Based on the characteristics and advantages of the above technologies, this study selects coal samples with different strengths, uses NMR technology to measure the initial cracks in coal samples to study the crack distribution and crack rate within the coal samples. Then, AE technology is used to detect the fracture development in the process of rock failure. By analyzing the ringing distribution and $\mathrm{AE}$ energy in the loading process, the crack development and failure mode are obtained. In addition, the key fracture that dominates the deformation and failure of the coal sample are restored by 3D-CAD. The distribution and development of coal fractures were studied by using NMR, AE and 3D-CAD restore technology, which provides a theoretical basis and testing method for the study of bearing characteristics, failure occurrence and energy accumulation of coal with different strengths.

\section{State of the art}

The macro-fracture of coal is important in its bearing capacity and failure mode. In the 1920s, it was found that the cracks changed its internal stress state, and the cracks could expand and penetrate under loading, resulting in material damage [5]. Hoek-Brown criterion was used to measure the 
strength of rock mass with cracks, and provides a method for the relationship between rock mass strength and cracks [6]. Ingraffea et al. studied the expansion of cracks and its influence on rock strength through uniaxial compression test. They proposed that the increase and penetration of internal cracks in rock samples are the reason for the final failure of rock [7-8]. Wang et al. prepared samples of the similar materials and prefabricated cracks, and conducted the seepage behaviour of fractured rock mass infilling using different transfixion rates under cyclic loading [9].

With regard to the research on the relationship between structure failure and dynamic disasters, Pan et al. analyzed the energy storage and energy consumption of coal and rock from the perspective of micro-structure and constructed a quantitative relationship between the lithologic fabric of the coal and rock mass and its impact tendency [10]. Zhao et al. used X-ray diffraction, SEM and automatic micro photometer to analyze the strength of coal impact tendency, and obtained the relationship between coal internal mesostructure parameters and impact tendency [11]. Jiang et al. used XRD, paramagnetic resonance, SEM to obtain the structural differences of coal and rock samples before and after rockburst, and explored the characteristics of energy dissipation in the process of rockburst incubation and occurrence [12]. Xue et al. found that the fractal reconstruction could describe the anisotropy effectively and the four roughness descriptors of asperity height, inclination angle, line length and surface area descriptors showed high precision for fractal dimension calculation [13]. In fact, coal is a typical soft rock, whereas its deformational behaviour is dominated by the two aspects: micro-structure and gas migration [14]. The structure of coal and rock is contributed to its geological conditions, including cleats and joints generated before and after formation of the coal and rock [15-19]. However, there are few studies on the influence of initial joint and fracture development on the deformation and failure characteristics and energy accumulation of coal and rock mass [20-23].

Therefore, this study will study the failure mode, strength and energy accumulation of coal and rock mass from the perspective of internal crack and fracture of coal and rock mass with different bearing capacity, and explore the essential relationship between deformation and failure and energy accumulation of the coal with different strengths to understand the mechanism of coal deformation and dynamic disasters in coal mines from the aspect of joints and fissures.

The rest of this study is organized as follows. Section 3 describes the samples and tesing procedure. Section 4 gives the experimental results and discussion. Finally, the conclusions are summarized in Section 5.

\section{Methodology}

\subsection{Sample preparation}

The testing samples are taken from Datong Xinzhouyao coal mine and Shuangya coal mine. The average compressive strength of the coal samples from Xinzhouyao coal mine is $30 \mathrm{MPa}$ and the average compressive strength of the coal samples from Dongrong No. 2 coal mine is $10 \mathrm{MPa}$. Two kinds of coal samples with different strengths are selected in the test to compare and contrast the influence of initial cracks, the deformation characteristics, failure modes and energy accumulation characteristics of coal.
After the coal sample was taken, it was processed by rock cutter and coring machine to $\phi 50 \mathrm{~mm} \times 100 \mathrm{~mm}$ standard rock mechanics testing specimen. The cross-section parallelism was controlled within $\pm 0.02 \mathrm{~mm}$. A total of 18 samples are prepared, 9 samples from each coal mine, as shown in Fig. 1. After the sample was prepared, it was distinguished according to the surface cracks, then the samples are scanned by NMR imaging technology to obtain the spatial distribution of cracks in the samples, and finally they are grouped according to different cracks.

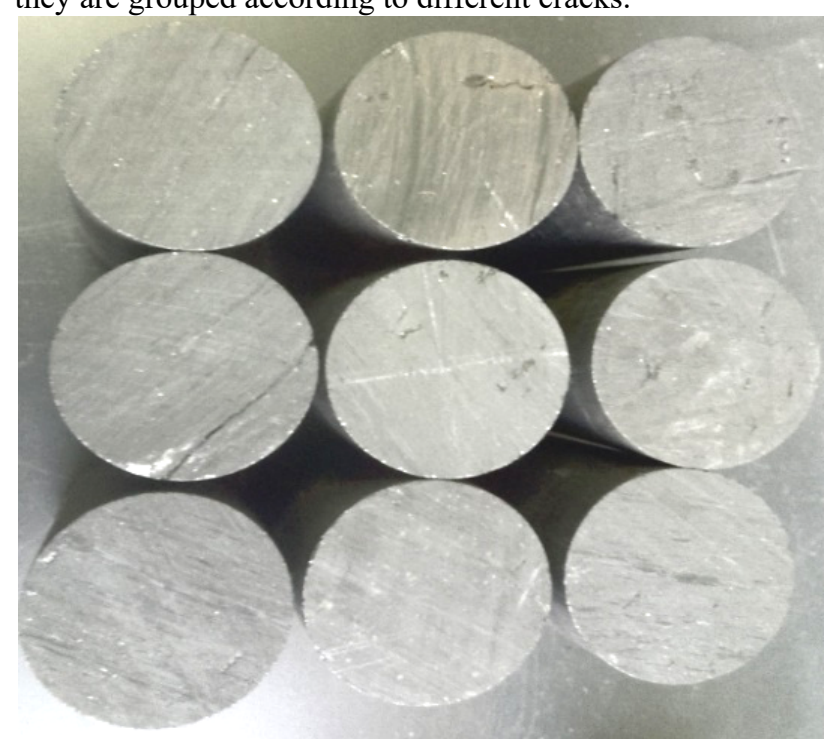

Fig. 1. Coal samples used in this study.

\subsection{NMR testing}

The NMR equipment is shown in Fig. 2(a). The applied intensity of the magnetic field is $0.3-0.5 \mathrm{~T}$, the main frequency of the instrument is $12 \mathrm{MHz}$. To ensure that the sample is in the middle of the magnetic field, the coil diameter of the magnetic field was selected as $60 \mathrm{~mm}$. As shown in Fig. 2(b), the analysis equipment is a nuclear magnetic imaging software and a gradient pulse instrument. The imaging software can set the slice width, number of slices and slice gap of the sample. After positioning, prescanning, formal scanning and imaging of the sample, the crack structure inside the sample is displayed in the form of slices.

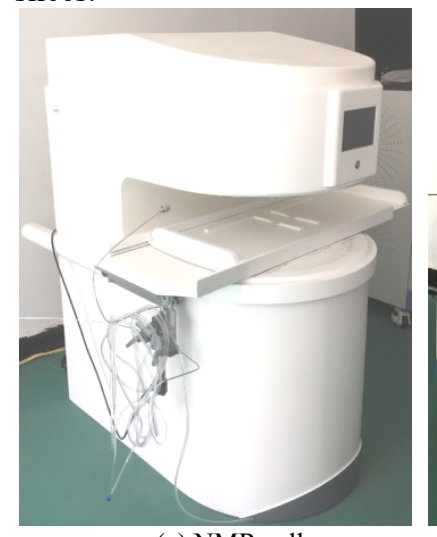

Fig. 2. NMR equipment used in this study

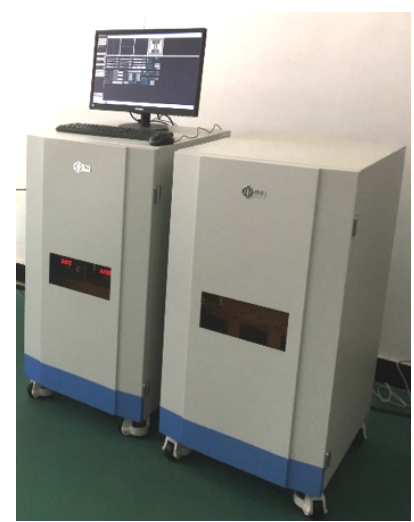

(b) NMR processing unit

The internal fracture distribution map of the sample can be obtained by processing the three-dimensional slice map using NMR 3D reconstruction software. The NMR 3D reconstruction map of typical samples in Xinzhouyao coal mine is shown in Fig. 3, in which the white areas indicate the fracture. 


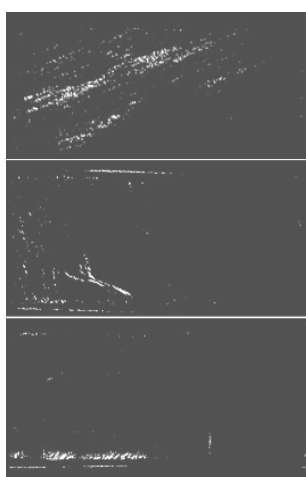

(a) Main view

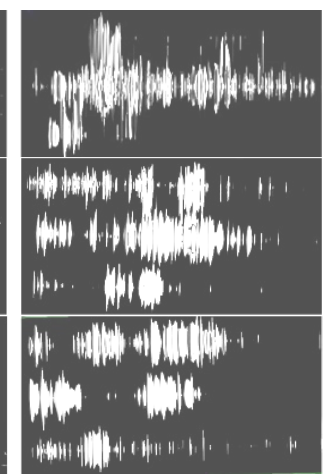

(b) Top view

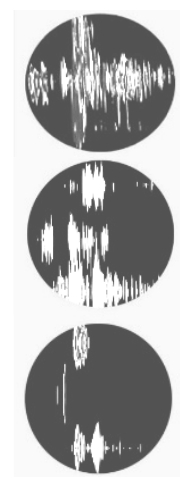

(c) Side view

Fig. 3. NMR reconstruction of Xinzhouyao coal mine samples

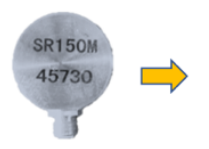

Sensor

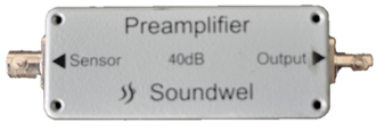

Amplifier

Fig. 4. Basic composition of acoustic emission system.

\subsection{Experimental Procedure}

WAW-600C computer-aided electro-hydraulic servo universal testing machine is used to conduct the compressive test. AE monitoring device is instrumented to observe the dynamic response of the specimen structure under uniaxial loading. The maximum loading capability is $600 \mathrm{kN}$, the maximum laoding velocity is $70 \mathrm{~mm} / \mathrm{min}$ and the maximum distance between the compression plates is $500 \mathrm{~mm}$.

The uniaxial compression testing system and the $\mathrm{AE}$ monitoring system are used to dynamically monitor the experimental data. The uniaxial compression testing machine is controlled by displacement with a loading rate of $0.5 \mathrm{~mm} / \mathrm{min}$. For parameters of the $\mathrm{AE}$ instrument, the threshold of the AE monitoring is set as $45 \mathrm{~dB}$ to minimize the effect of surrounding noises. The peak definition time (PDT), the hit definition time (HDT), and the hit locking time (HLT) are selected as $300 \mu \mathrm{s}, 600 \mu \mathrm{s}$, and $1000 \mu \mathrm{s}$, respectively. The sound velocity is set to be $1.8 \mathrm{~km} / \mathrm{s}$.

After parameter setting was completed, fusing test was conducted on the specimen to verify the sensitivity of each sensor to guarantee the reliability of the experiment. After the parameter setting and fusing testing are completed, uniaxial compression tests are carried out. The stress loading system and the $\mathrm{AE}$ monitoring system were turned on simultaneously. The experimental arrangement and the posttesting samples are shown in Fig. 5.

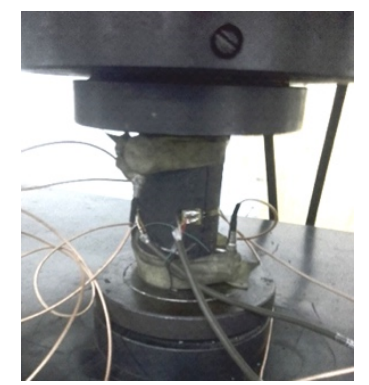

(a) Before

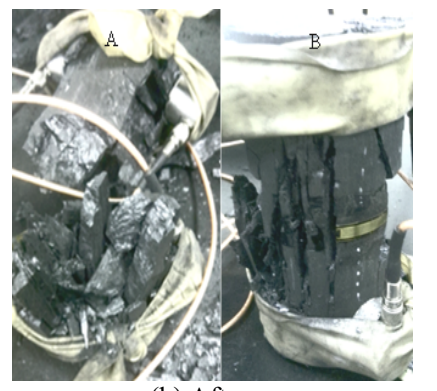

(b) After

Fig. 5. Before and after testing samples

\subsection{AE testing}

The AE technology is an effective method to detect the dynamic change of the material structure under the action of external loading through instrumentation. Each channel of the data acquisition system consists of measuring instrument, digital signal processor, computation program and other peripheral apparatus, which are finally connected to a computer. The components of each channel include an AE sensor, a preamplifier, and a data acquisition card, as shown in Fig. 4.

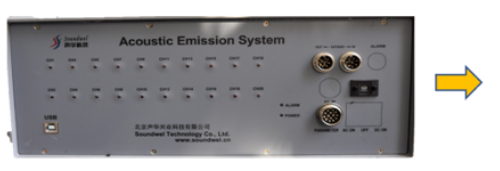

Data acquisition

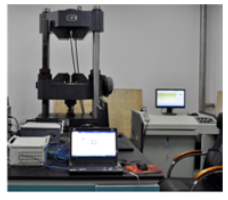

Computer

\section{Results and Discussion}

\subsection{The initial crack rate and distribution}

According to the NMR data, the initial crack rate can be calculated. As the internal crack of the sample is an irregular body, to simply the calculation, the internal crack of the sample is regarded as a regular cuboid, and the parameter index is length, width and height. Table 1 shows the specific parameters of the internal crack of the coal samples. The sample number of Xinzhouyao coal mine starts with $\mathrm{X}$ and that of Dongrong No. 2 coal mine starts with D. the crack rate of the coal sample is calculated, as shown in Table 2.

By import the $3 \mathrm{D}$ slice diagram of the sample into $\mathrm{CAD}$, the $\mathrm{CAD} 3 \mathrm{D}$ reconstruction diagram shows the fracture development and parameters. The CAD three-dimensional reconstruction diagram is shown in the Fig. 6.

\subsection{Crack development and distribution}

The AE detection threshold was set to $45 \mathrm{~dB}$ and the sound speed is $1.8 \mathrm{~km} / \mathrm{s}$. Fig. 7 shows the representative AE fracture point of coal sample and the corresponding CAD reconstruction diagram.

It can be seen that the original fracture exists at the first position of the fracture point. With the progress of loading, the original fracture begins to expand and penetrate until the sample is damaged. During this period, the AE fracture point increases with the fracture, and the positions of fracture points are the positions of fracture expansion. Comparing X1 and $\mathrm{X} 2$, in which $\mathrm{X} 1$ fracture rate is $0.06 \%$ and $\mathrm{X} 2$ fracture rate is $0.18 \%$, it can be seen that the higher the fracture rate is, the earlier the fracture point appears. Comparing X2 sample with different strengths with D2, X2 fracture rate is $0.18 \%, \mathrm{D} 2$ fracture rate is $0.17 \%$, and the difference of fracture rate is only $0.01 \%$, which can be ignored. However, the occurrence time of $\mathrm{X} 2$ fracture point of sample is 3 times that of D2 fracture point. Therefore, the greater the strength, the later the occurrence time of fracture point of sample. From the simple analysis of the total stress-strain curve of uniaxial compression, the stage of fracture points is the compaction stage, and the time stage of a large number of fracture points is the middle stage of linear elasticity stage. 
Table. 1 The initial crack of each coal sample

\begin{tabular}{|c|c|c|c|c|c|c|}
\hline \multirow{2}{*}{ Name } & \multirow{2}{*}{ No. } & \multirow{2}{*}{ Position } & \multicolumn{4}{|c|}{ Dimension } \\
\hline & & & Direction & Length (mm) & Width (mm) & Thickness (mm) \\
\hline X1-1 & 1 & Top $5.2 \mathrm{~mm}$ & Horizontal & 23.8 & 13.2 & 0.25 \\
\hline$X 1-2$ & 1 & Bottom $12.3 \mathrm{~mm}$ & Horizontal $30^{\circ}$ & 25.8 & 9.5 & 0.96 \\
\hline \multirow{2}{*}{ X1-3 } & 1 & Bottom 38 mm & Horizontal $45^{\circ}$ & 27.6 & 13.8 & 0.62 \\
\hline & 2 & inclined & Vertical & 25.3 & 19.3 & 0.78 \\
\hline X2-1 & 1 & Bottom $5 \mathrm{~mm}$ & Horizontal & 19.2 & 17 & 0.36 \\
\hline \multirow{3}{*}{$\mathrm{X} 2-2$} & 1 & Top $45 \mathrm{~mm}$ & Horizontal & 33.1 & 16.4 & 0.40 \\
\hline & 2 & Top & Vertical & 24.3 & 13.2 & 0.42 \\
\hline & 1 & Top $55 \mathrm{~mm}$ & Horizontal & 19.5 & 17.9 & 1.00 \\
\hline \multirow[t]{2}{*}{$X 2-3$} & 2 & Top $50 \mathrm{~mm}$ & Horizontal & 27.4 & 16.3 & 0.3 \\
\hline & 3 & Top $10 \mathrm{~mm}$ & Vertical & 21.3 & 10.1 & 0.22 \\
\hline X3-1 & 1 & Top-middle & Vertical & 38.3 & 15.3 & 0.1 \\
\hline \multirow{3}{*}{ X3-2 } & 1 & Top-middle & Vertical & 48.3 & 9.3 & 0.3 \\
\hline & 2 & Middle & Horizontal $80^{\circ}$ & 30.1 & 18.2 & 0.25 \\
\hline & 1 & Bottom $10 \mathrm{~mm}$ & Horizontal & 26.3 & 16.1 & 0.5 \\
\hline \multirow[t]{2}{*}{ X3-3 } & 2 & Bottom $20 \mathrm{~mm}$ & Horizontal & 28.4 & 15.0 & 0.5 \\
\hline & 3 & Bottom $35 \mathrm{~mm}$ & Horizontal & 23.4 & 21.5 & 0.52 \\
\hline D1-1 & 1 & Bottom $20 \mathrm{~mm}$ & Horizontal $60^{\circ}$ & 42.3 & 8.4 & 0.55 \\
\hline \multirow{2}{*}{ D1-2 } & 1 & Middle-bottom & Vertical & 38.7 & 10.0 & 0.77 \\
\hline & 2 & Bottom 40mm & Horizontal $45^{\circ}$ & 23.6 & 13.1 & 0.68 \\
\hline \multirow{4}{*}{ D1-3 } & 1 & Bottom $45 \mathrm{~mm}$ & Horizontal & 24.7 & 17.6 & 0.40 \\
\hline & 2 & Bottom & Horizontal $60^{\circ}$ & 41.7 & 18.2 & 0.32 \\
\hline & 3 & Top & Vertical & 38.0 & 21.4 & 0.41 \\
\hline & 4 & Top & Vertical & 46.8 & 13.9 & 0.60 \\
\hline D2-1 & 1 & Bottom $46 \mathrm{~mm}$ & Horizontal & 35.2 & 18.3 & 0.52 \\
\hline \multirow{2}{*}{ D2-2 } & 1 & Bottom $33 \mathrm{~mm}$ & Horizontal $30^{\circ}$ & 22.0 & 18.2 & 1.13 \\
\hline & 2 & Top & Vertical & 42.3 & 7.2 & 0.71 \\
\hline \multirow{3}{*}{ D2-3 } & 1 & Bottom $27 \mathrm{~mm}$ & Vertical & 22.5 & 17.8 & 0.63 \\
\hline & 2 & Bottom & Horizontal $50^{\circ}$ & 32.3 & 28.7 & 0.69 \\
\hline & 3 & Top & Vertical & 26.5 & 22.5 & 0.4 \\
\hline \multirow{2}{*}{ D3-1 } & 1 & Top 30mm & Horizontal & 27.4 & 19.8 & 0.48 \\
\hline & 2 & Bottom $42 \mathrm{~mm}$ & Horizontal $55^{\circ}$ & 24.1 & 18.4 & 0.63 \\
\hline \multirow{3}{*}{ D3-2 } & 1 & Bottom & Vertical & 50.0 & 17.0 & 0.42 \\
\hline & 2 & Bottom $24 \mathrm{~mm}$ & Horizontal $40^{\circ}$ & 23.4 & 21.4 & 0.73 \\
\hline & 3 & Top & Vertical & 52.0 & 23.8 & 0.31 \\
\hline \multirow{4}{*}{ D3-3 } & 1 & Bottom $13 \mathrm{~mm}$ & Horizontal $60^{\circ}$ & 64.7 & 15.2 & 1.2 \\
\hline & 2 & Bottom & Vertical & 51.3 & 22.4 & 0.28 \\
\hline & 3 & Top $15 \mathrm{~mm}$ & Horizontal & 18.4 & 16.7 & 0.4 \\
\hline & 4 & Top $17 \mathrm{~mm}$ & Vertical & 23.4 & 8.4 & 0.23 \\
\hline
\end{tabular}

Table. 2 Basic information and fracture rate of coal samples

\begin{tabular}{|c|c|c|c|c|}
\hline \multirow{2}{*}{ Name } & \multirow{2}{*}{ No. } & \multicolumn{2}{|c|}{ Dimension } & \multirow{2}{*}{ Crack rate } \\
\hline & & Diameter (mm) & Height (mm) & \\
\hline \multirow{9}{*}{$\mathrm{X}$} & $\mathrm{X} 1-1$ & 50.14 & 100.84 & $0.04 \%$ \\
\hline & $\mathrm{X} 1-2$ & 50.21 & 100.10 & $0.12 \%$ \\
\hline & $\mathrm{X} 1-3$ & 50.11 & 100.03 & $0.32 \%$ \\
\hline & $\mathrm{X} 2-1$ & 50.17 & 100.05 & $0.06 \%$ \\
\hline & $\mathrm{X} 2-2$ & 50.12 & 100.63 & $0.18 \%$ \\
\hline & $X 2-3$ & 50.08 & 100.42 & $0.27 \%$ \\
\hline & X3-1 & 50.04 & 100.04 & $0.03 \%$ \\
\hline & X3-2 & 50.17 & 100.84 & $0.14 \%$ \\
\hline & X3-3 & 50.02 & 100.45 & $0.35 \%$ \\
\hline \multirow{9}{*}{$\mathrm{D}$} & D1-1 & 50.08 & 100.51 & $0.10 \%$ \\
\hline & D1-2 & 50.10 & 100.08 & $0.26 \%$ \\
\hline & D1-3 & 50.03 & 100.77 & $0.58 \%$ \\
\hline & D2-1 & 50.07 & 100.25 & $0.17 \%$ \\
\hline & D2-2 & 50.13 & 100.15 & $0.33 \%$ \\
\hline & D2-3 & 50.05 & 100.51 & $0.58 \%$ \\
\hline & D3-1 & 50.04 & 100.45 & $0.28 \%$ \\
\hline & D3-2 & 50.13 & 100.09 & $0.55 \%$ \\
\hline & D3-3 & 50.08 & 100.11 & $0.84 \%$ \\
\hline
\end{tabular}



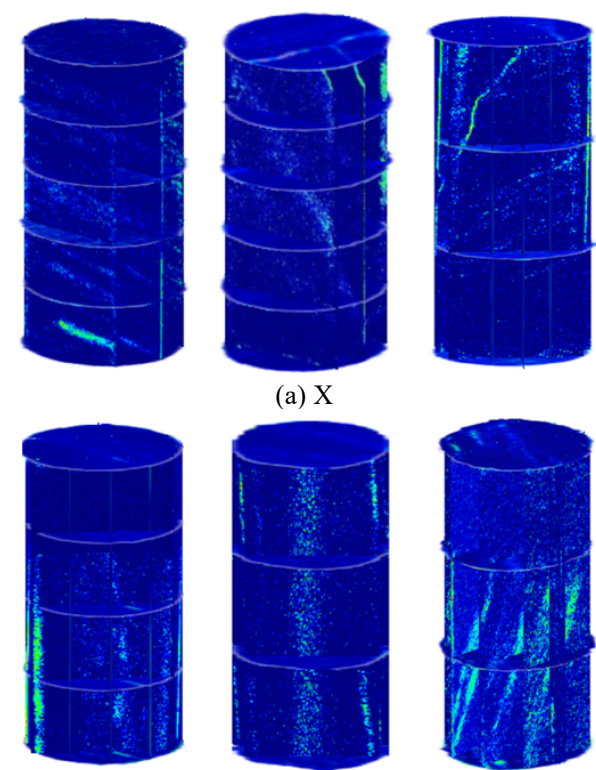

(a) $X$
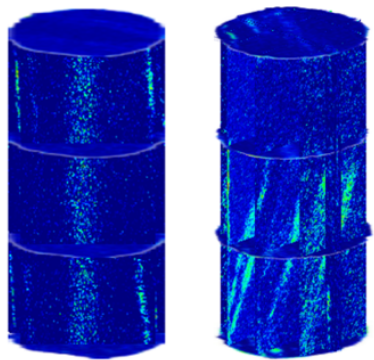

(b) D
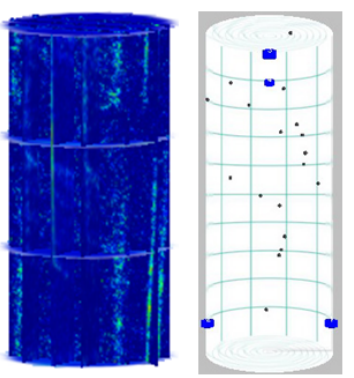

$30 \mathrm{~s}$

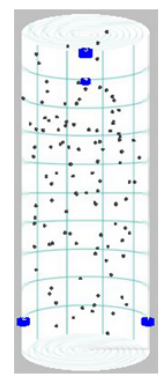

$60 \mathrm{~s}$

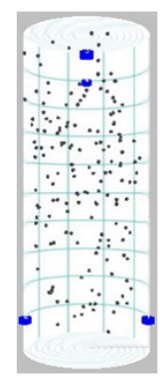

$100 \mathrm{~s}$

(d) D2

Fig. 7. AE fracture point and the corresponding CAD reconstruction diagrams of X1, X2, D1, and D2.

\subsection{Ring accounts and uniaxial strength}

By processing the $\mathrm{AE}$ data, the ring account and stress and energy diagram of each sample are obtained. The stress is obtained from the uniaxial compression data, and the ringing count and energy are detected by the AE instrument. Typical diagram is shown in Fig. 8.
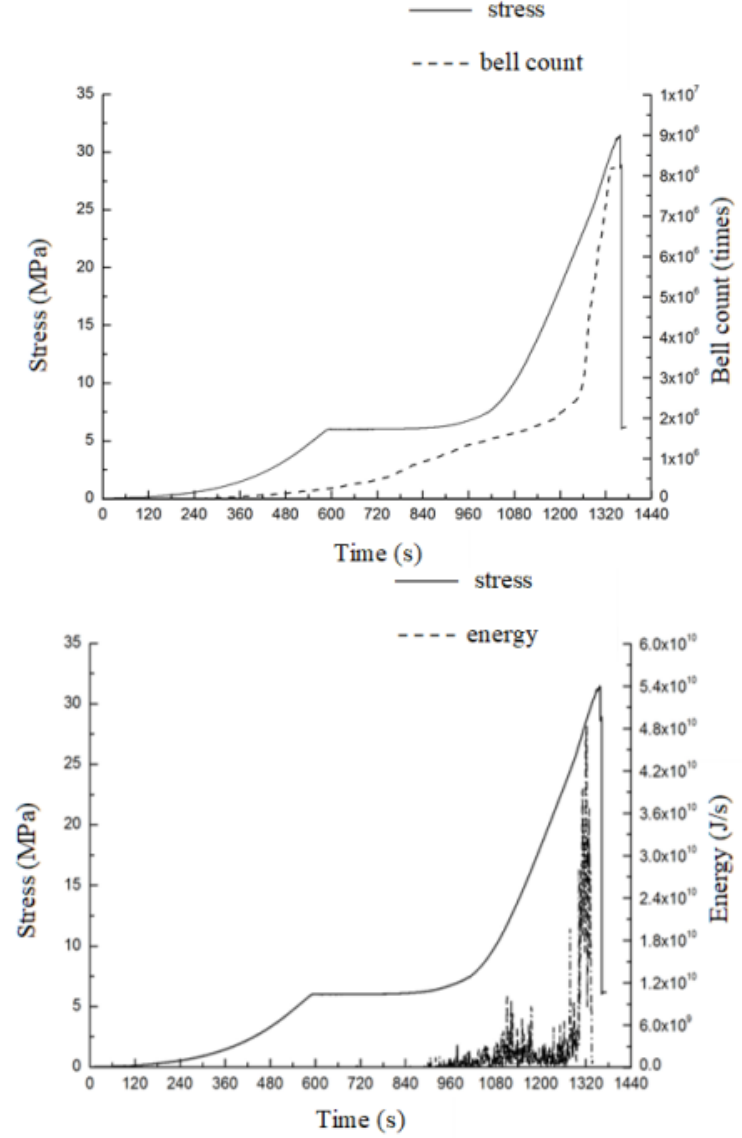

(a) X1
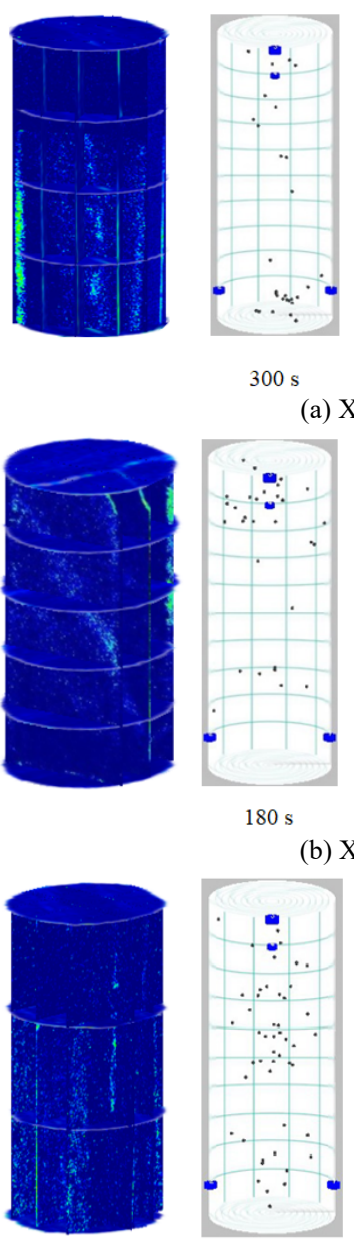

$300 \mathrm{~s}$

(a) X1
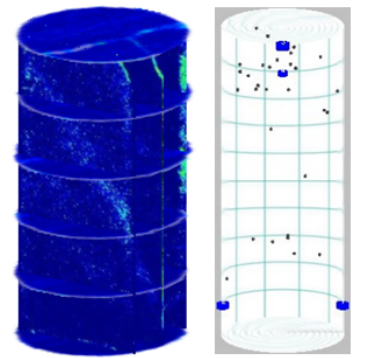

$180 \mathrm{~s}$

(b) X2

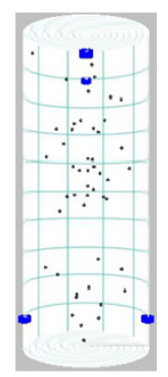

$60 \mathrm{~s}$

(c) D1

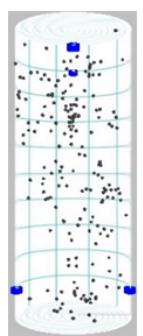

$360 \mathrm{~s}$

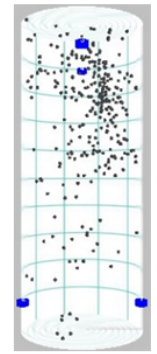

$360 \mathrm{~s}$

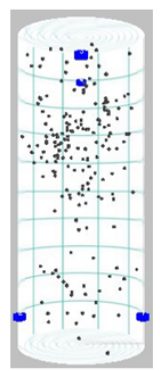

$100 \mathrm{~s}$

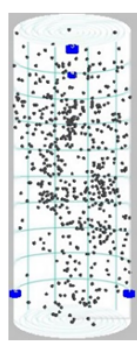

$480 \mathrm{~s}$

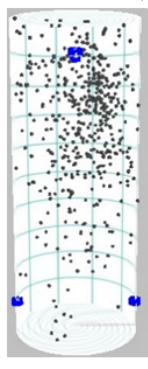

$540 \mathrm{~s}$

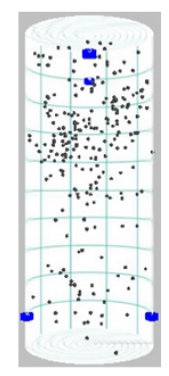

$20 \mathrm{~s}$ 

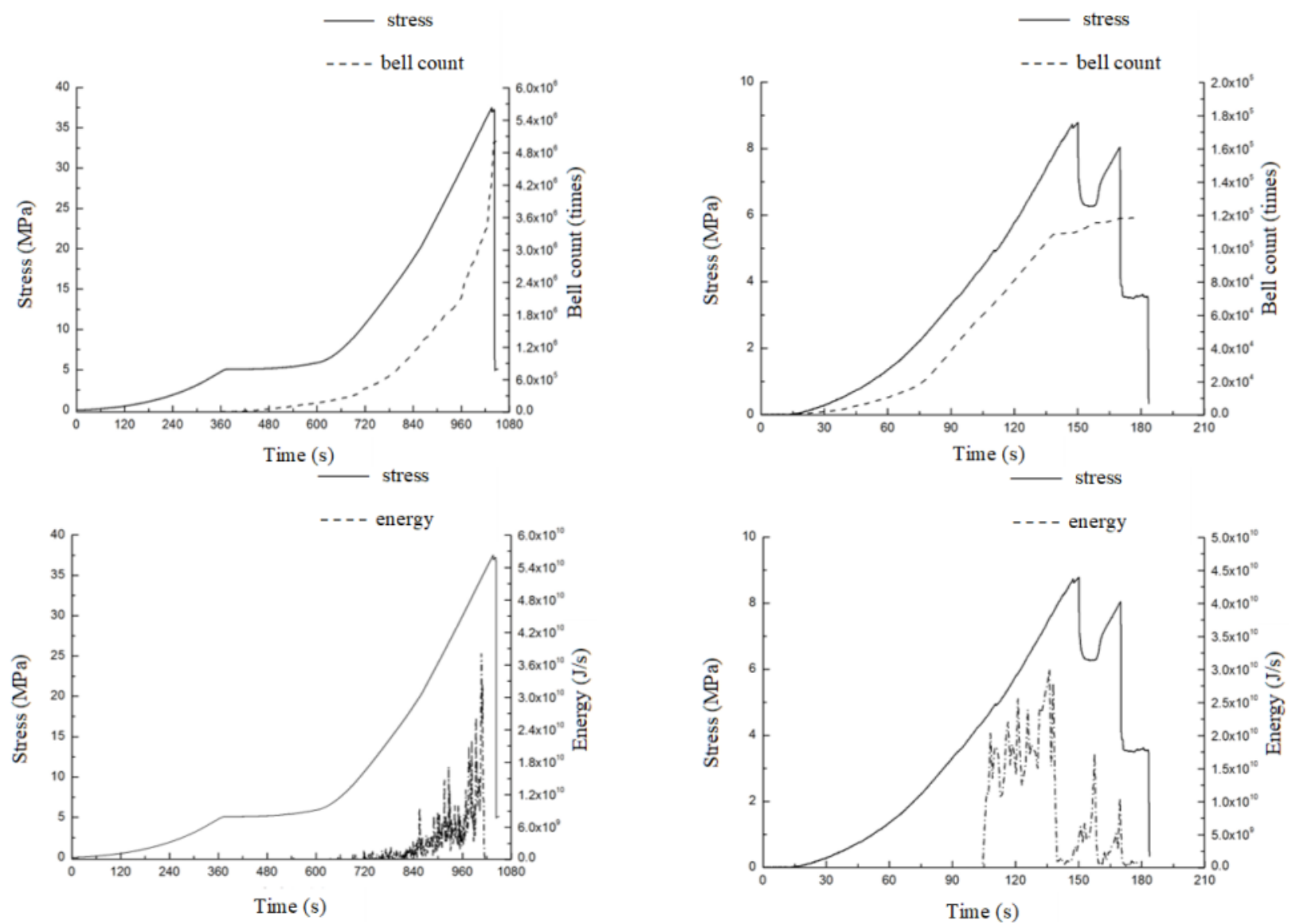

(b) X2 stress
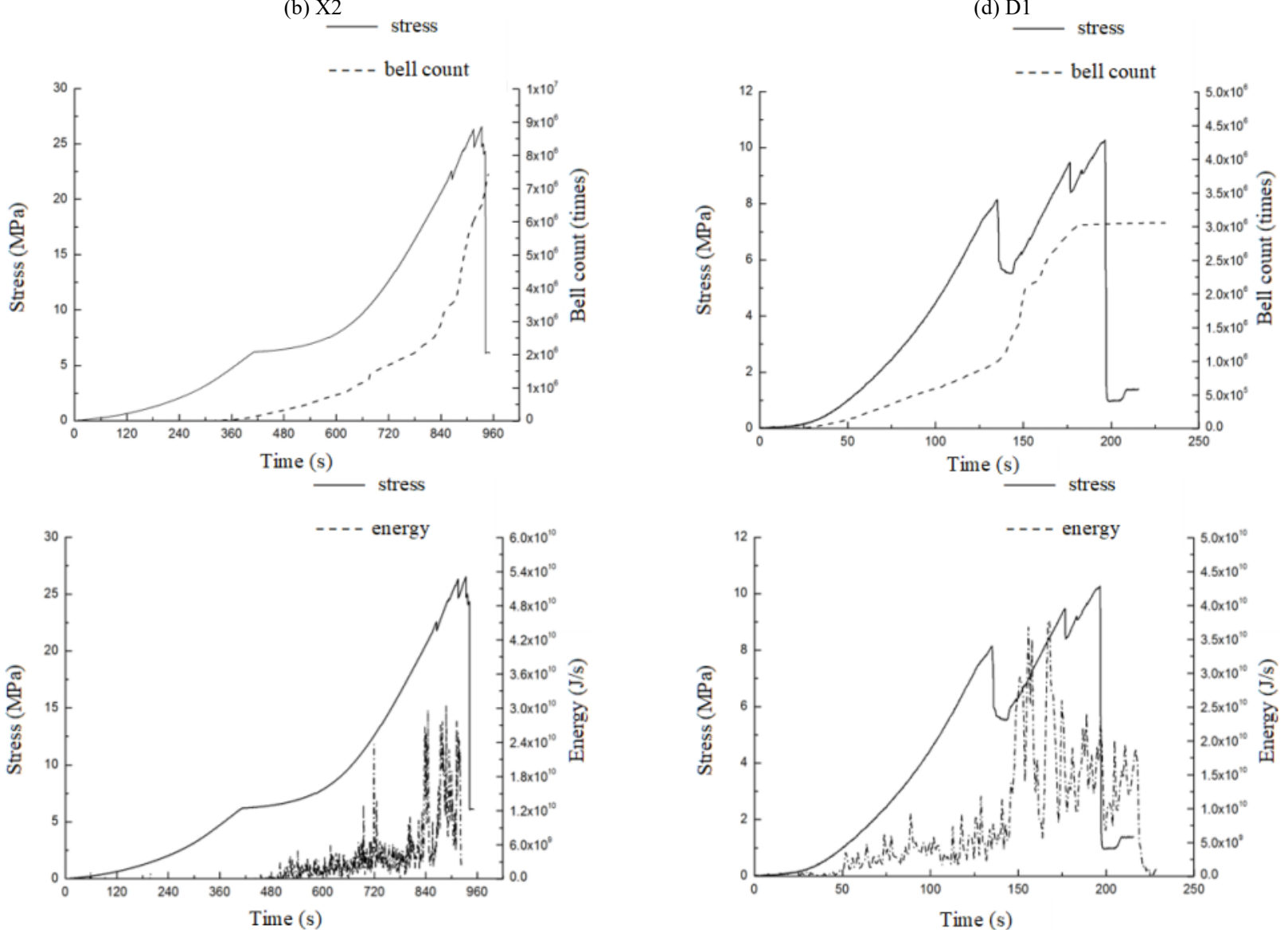

(c) $\mathrm{X} 3$ 

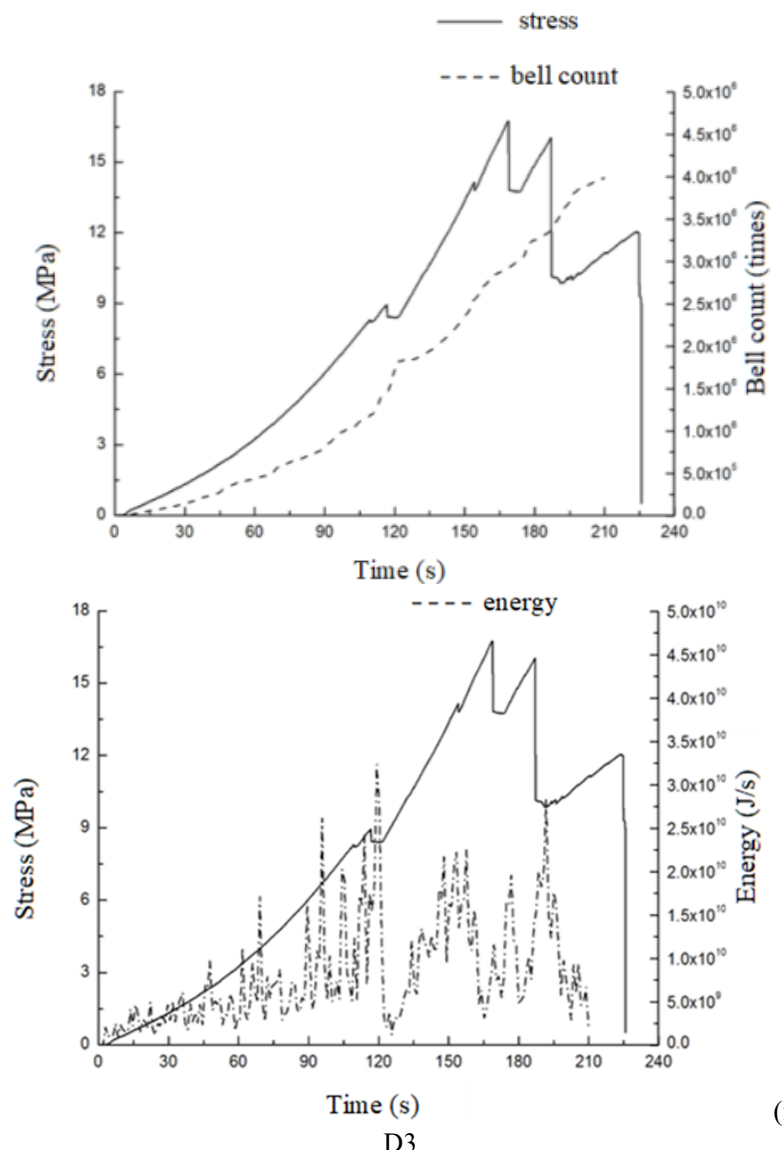

(f)

Fig. 8. Ring accounts and energy of $\mathrm{AE}$ of $\mathrm{X} 1, \mathrm{X} 2, \mathrm{X} 3, \mathrm{D} 1, \mathrm{D} 2$, and $\mathrm{D} 3$.

The ringing count in the is the number of times that the shock wave generated when the sample is over the detection threshold. The more the ringing count, the more the internal damage of the sample. The energy diagram is not the real energy released by the specimen damage, but the AE records the output in the form of corresponding energy value.

$\mathrm{X} 1$ sample with the smallest crack number and crack rate has almost no ring count within $360 \mathrm{~s}$ at the initial stage of loading. After that, the ring count increases slowly until the ring count suddenly rises straight in the linear elastic stage, and the total number reaches $8.2 \times 106$ times. The ring count of X2 sample with relatively large crack number and crack rate is very similar to that of $\mathrm{X} 3$ sample, which is relatively gentle compared with $\mathrm{X} 1$ sample, and the ring count reaches $5.1 \times 106$ times and $6.5 \times 106$ times. As there are many primary cracks in X2 and X3 samples, so the failure mode of them is slow expansion and penetration of multiple cracks until failure. The X1 sample has fewer internal cracks, therefore, the failure of the sample is the sudden occurred, and the ring count shows a trend of slowly increasing to straight rising.

The stress energy diagram of $\mathrm{X}$ samples shows that, the energy curve trend of X1 sample with low fracture rate is consistent with its ring count trend. It also shows a straight rise from the slow increase in the initial stage of loading to the linear elastic stage, which is consistent with the failure characteristics of less cracks in the sample, resulting in secondary cracks and rapid expansion. The final energy peak is $4.8 \times 1010 \mathrm{~J} / \mathrm{s}$. The energy curve trend of X2 and X3 samples with high fracture rate is also consistent with their failure characteristics. They show the trend that the energy value increases slowly in the initial stage of loading until a small increase in the linear elastic stage, and their energy peaks are $3.8 \times 1010$ and $3.1 \times 1010 \mathrm{~J} / \mathrm{s}$, respectively.
The average compressive strength of the $\mathrm{D}$ sample is only $10 \mathrm{MPa}$. The coal is soft and the fracture rate is higher than that of $\mathrm{X}$. Therefore, the law of ring count curve and energy value curve is different from that of $X$. The ring counts of the three samples appear at the initial stage of stress loading, and the ring counts gradually increase with the progress of loading, and the ring counts are $1.2 \times 105$, $3.1 \times 106$ and $4.0 \times 106$ respectively. It can be seen that the failure characteristics of coal samples with more cracks are the joint expansion and penetration of multiple cracks in the sample and the slow increase of secondary cracks until the sample is damaged. In terms of stress energy diagram, D1 sample with the lowest crack rate has no energy value at the initial stage of loading, and the energy value increases slightly at the online elastic stage, with a peak value of $3.0 \times$ $1010 \mathrm{~J} / \mathrm{s}$. D2 and D3 samples with high fracture rate have energy values from the initial stage of stress loading, and then gradually increase, with peak values of $3.8 \times 1010$ and $3.3 \times 1010 \mathrm{~J} / \mathrm{s}$, respectively.

\subsection{Failure process of fractured coal mass}

Through the analysis of the NMR and AE test results of coal samples in, it can be seen that the crack is the main factor affecting the failure characteristics and energy accumulation for coal samples. The ring count and energy peak stage of coal samples are statistically analyzed, as shown in Table 3.

Table. 3 Failure process

\begin{tabular}{c|c|c|c|c}
\hline Name & No. & Crack & Ring account & Peak energy \\
& X1-1 & $0.04 \%$ & Ealy elastic & Later elastic \\
& X1-2 & $0.12 \%$ & Later compaction & Later elastic \\
X1-3 & $0.32 \%$ & Ealy compaction & Ealy elastic \\
& X2-1 & $0.06 \%$ & Middle elastic & Peak \\
X & X2-2 & $0.18 \%$ & Later compaction & Later elastic \\
& X2-3 & $0.27 \%$ & Later compaction & Ealy elastic \\
& X3-1 & $0.03 \%$ & Middle compaction & Peak \\
& X3-2 & $0.14 \%$ & Middle compaction & Peak \\
& X3-3 & $0.35 \%$ & Ealy compaction & Later elastic \\
\hline \multirow{6}{*}{ D $1-1$} & $0.10 \%$ & Ealy compaction & Later elastic \\
& S1-2 & $0.26 \%$ & Ealy compaction & Residual \\
& S1-3 & $0.58 \%$ & Ealy compaction & Later \\
& S2-1 & $0.17 \%$ & Middle elastic & Later elastic \\
& S2-2 & $0.33 \%$ & Later compaction & Peak \\
& S2-3 & $0.58 \%$ & Ealy compaction & Ealy compaction \\
& S3-1 & $0.28 \%$ & Ealy elastic & Peak \\
& S3-2 & $0.55 \%$ & Later compaction & Later elastic \\
& S3-3 & $0.84 \%$ & Ealy compaction & Middle elastic \\
\hline
\end{tabular}

As shown in Table 3, due to the different fracture rates of each coal sample, the ring count and energy peak are also different. It can be seen from the analysis that the internal cracks of the sample increase with the loading, and the stages of ring count and energy peak are different. On the other hand, due to the increase of cracks in the sample, the accumulated energy of the sample is released in advance, and the time of energy accumulation of the sample becomes shorter.

\section{Conclusions}

In this study, from the point of view of the initial cracks distribution and crack rate of the sample, the influence of the fracture development of the coal sample with different on the failure characteristics and energy accumulation is studied 
using NMR, EA and CAD-3D restoration technologies. Following conclusions can be obtained:

(1) The number of cracks and its parameters in coal samples are quantified by measurement and calculation by the NMR imaging technology and CAD-3D reconstruction, which are dominated factors for fracture development under loading conditions;

(2) Through the uniaxial compression test, acoustic emission properties are obtained to study the failure mode of coal samples with different fracture rate. Results shows that sample with high compressive strength are manifested in the expansion and penetration of the internal primary fractures; and that of the sample with low compressive strength derived secondary fractures, for which, the ring count curve shows a slow upward trend;
(3) The failure mode of samples with low fracture rate are that with the gradual loading to the linear elastic stage, a large number of secondary fractures are rapidly derived from the existing primary fractures, so that the ring count curve suddenly rises straight from the linear elastic stage;

(4) In terms of AE energy accumulation, by drawing the curves of fracture number, fracture rate and energy peak, it is concluded that the energy of samples with higher fracture number and fracture rate is released in advance and the energy peak is reduced.

\section{Acknowledgements}

This work was financially supported by the The National Natural Science Fund of China (5177041704).

\section{References}

1. Li, Z. C., Li, L. C., Wang, S. R., Ma, S., Zhang, Z. L., Li, A. S., Huang, B., Zhang, L. Y., Wang, Z. L., Zhang, Q. S., "Formation of $\mathrm{X}$-shaped hydraulic fractures in deep thick glutenite reservoirs: A case study in Bohai Bay Basin, East China”. Journal of Central South University, 27(10), 2020, pp. ID 0397.

2. Xue, D. J., Zhou, H. W., Liu, Y. T. Deng, L. S., Zhang, L. "Study of drainage and percolation of nitrogen-water flooding in tight coal by NMR imaging," Rock Mechanics and Rock Engineering, 51(11), 2018, pp. 3421-3437.

3. Dou, L., Yang, K, Chi, X. "Fracture behavior and acoustic emission characteristics of sandstone samples with inclined precracks". International Journal of Coal Science \& Technology, 8, 2021, pp. 77-87.

4. Xue, D. J., Lu, L. L., Zhou, J., Lu, L., Liu, Y. T., "Cluster modeling of the short-range correlation of acoustically emitted scattering signals". International Journal of Coal Science \& Technology, 22, 2020, pp. 1-15.

5. Jamali, J., Naei, M. H., Honarvar, F., Rajabi, M., “Acoustic scattering and radiation force function experienced by functionally graded cylindrical shells". Journal of Mechanics, 27(2), 2011, pp.227-243.

6. Jiang, F. X., Shu, C. X., Wang, C. W., "Impact risk assessment of mining face based on stress superposition". Journal of Rock Mechanics and Engineering, 34 (12), 2015, pp. 2428-2435.

7. Ingraffea, A. R., Heuze, F. E., "Finite element models for rock fracture mechanics". International Journal for Numerical \& Analytical Methods in Geomechanics, 4(1), 1980, pp. 25-43.

8. Palmstrom, A., "Characterizing rock masses by the RMi for use in practical rock engineering: Part 1: The development of the Rock Mass index (RMi)". Tunnelling \& Underground Space Technology, 11(2), 1996, pp. 175-188.

9. Wang, S. R., Zhang, J. Y., Li, Z. C., Yu, Y. Q., "Seepage behaviour of fractured rock mass infilling using different transfixion rates under cyclic loading”. Geofluids, 2021, 2021, pp. ID 9995090.

10. Pan, J. N., Meng, Z. P., Liu, B. M., "The composition and structure of coal rock and its impact tendency". Journal of Rock Mechanics and Engineering, 24 (24), 2005, pp. 4422-4427.

11. Zhao, Y. X., Jiang, Y. D., Zhang, Y., "Relevant law of impact tendency and fine observation structure characteristics of coal body". Journal of Coal, 32 (1), 2007, pp. 64-68.

12. Jiang, Y. D., Lv, Y. Q., Zhao, Y. X., Song, Y. M., Tao, L., "Multiparameter monitoring test for coal sample instability failure". Journal of Rock Mechanics and Engineering, 31(4), 2012, pp. 667674.
13. Xue, D. J., Liu, Y. T., Zhou, H. W., Wang, J. Q., Zhou, J., "Fractal characterization on anisotropy and fractal reconstruction of rough surface of granite under orthogonal shear," Rock Mechanics and Rock Engineering, 53(3), 2020, pp. 1225-1242.

14. Mao, S. J., "Development of coal geological information technologies in China". International Journal of Coal Science \& Technology, 7, 2020, pp. 320-328.

15. Zhao, Y. H.,, Wang, S. R., Zou, Z. S., Ge, L. L., Cui, F., "Instability characteristics of the cracked roof rock beam under shallow mining conditions". International Journal of Mining Science and Technology, 28(3), 2018, pp. 437-444.

16. Luo, P. P., Wang, S. R., Hagan, P., Huang, Q. X., Cao, C., Gamage, $\mathrm{K}$., "Influence of weak interlayer on mechanical performances of rock-like materials". Dyna, 94(4), 2019, pp. 447-454.

17. Wang, S. R., Wu, X. G., Zhao, Y. H., Hagan, P., Cao, C., "Evolution characteristics of composite pressure-arch in thin bedrock of overlying strata during shallow coal mining". International Journal of Applied Mechanics, 11(3), 2019, pp. ID 1950030 .

18. Zhao, Y. H., Wang, S. R., Zou, Y. F., Wang, X. C., Huang, B. Q., Zhang, X. G., "Pressure-arching characteristics of fractured strata structure during shallow horizontal coal mining". Technical Gazette, 25(5), 2018, pp. 1457-1466.

19. Drapaluk, M., "Influence of reinforced concrete forming features on mechanical characteristics". Technical Journal, 13(2), 2019, pp. 8691.20. Chang, X., Lu, J. Y., Wang, S. Y., Wang, S. R., Liu, J., "Formation of cracks in layered rock considering layer thickness variations". Geophysical Journal International, 210(3), 2017, pp. $1623-1640$.

21. Wang, S. R., Li, N., Li, C. L., Zou, Z. S., Chang, X., "Instability mechanism analysis of pressure-arch in coal mining field under different seam dip angles". Dyna, 90(3), 2015, pp. 279-284.

22. Chang, X., Deng, Y., Li, Z. H., Wang, S. R., Tang. C. A., "Crack propagation from a filled flaw in rocks considering the infill influences". Journal of Applied Geophysics, 152, 2018, pp. 137149 .

23. Wang, S. R., Zhao, J. Q., Wu, X. G., Yang, J. H., Liu, A., "Mesoscale simulations of lightweight aggregate concrete under impact loading". International Journal of Simulation Modelling, 20(2), 2021, pp. 291-302. 